\title{
Abstracts.
}

\section{NOSE, Etc.}

Avellis (Frankfurt).- The Importance of Ulceration of the Mucous Membrane in Acute Empyema of the Nasal Accessory Simuses. “Archiv. für Laryngologie und Rhinologie," Bd. 11, H. 3.

Spontaneous recovery is the rule in acute inflammation of the accessory sinuses. Where this does not occur, the cause is usually some obstruction to the free escape of discharge, and with its removal there is healing.

Two cases of empyema of the frontal sinus, with exceptionally severe pain, which increased with the duration of the empyema, and where no improvement was obtained by intranasal treatment, were operated on in the acute stage. Ulceration of the posterior wall, with exposed superficial necrosis of bone, was found in both cases, which explained the occurrence of non-spontaneous recovery. Gillil.

B. Fraenkel (Berlin).--Hyperestriesia of the Nasal Mucous Memlirane. "Berliner Klinische Wochenschrift," 1901, No. 15.

With reference to his former work on nasal reflex neuroses, Fraenkel emphasizes the important role which changes in the nasal nerves play. To demonstrate hyperæsthesia, liquor ammonia is used as well as a probe. A positive result is only obtained by the former in many cases. Surgical treatment of nasal reflex neurosis should be limited. He prefers cocaine as a means of overcoming the sensibility of the nasal mucous membrane, but he has also had good results from the use of orthoform. Of internal medicines, he places the salts of bromide first, with which he has had excellent results in hay fever.

\section{Guild.}

De Rosa Michele, Dr., Med. Capt. (Padua).-Rhinitis Caseosa. "Archiv. Ital. di Otologia," Torino, April to June, 1901.

The author describes three cases of this disease occurring in the clinic of Professor Arslan, who prefers the term rhinorrhe't purulenta caseiforma. In two cases there was empyema of the maxillary sinus, while in the third a large rhinolith was embedded in the cheesy mass.

Dr. Nichele refers to the various etiological theories of this affection, and discusses at considerable length the specific theory of Cozzolino, that the scrofulous diathesis and some microbe, specific or otherwise, acting especially on the epithelium are necessary, as well as the common theory that the caseous masses are the result of retention, and that the disease should be regarded as an epiphenomenon of other affections (foreign bodies, tumours, chronic sinusitis). Cozzolino being unable to set aside the great number of facts in favour of the common view, hints at the possibility of two forms of caseous rhinitis, the trut and the false. This view was supported by Guarnaccia in 1896 in a paper ${ }^{1}$ in which he described the Streptothrix alba as the specific microbe of the true affection, while it was absent from other forms. Dr. Michele has collected 49 cases from the literature: True caseous rhinitis, 9 ; pseudo-rhinitis caseosa, 40, the latter divided etiologically as follows-sinusitis or rhinitis purulenta, 14 ; rhinoliths, polypi and

\footnotetext{
1 Archie. Ital. di Laring., 1896.
} 
other tumours, 14 ; cause not stated, 12. The nine cases of so-called true caseous rhinitis were those in which there was no evident cause in the history, and which were therefore believed to depend on the scrofulous diathesis and a specific micro-organism. It is generally argued that in view of the prevalence of the scrofulous diathesis the number of cases of caseous rhinitis ought to be much greater if it depended on that cause. Masini, ${ }^{1}$ on the other hand, endeavours to turn this against the common theory by saying that, in view of the great frequency of nasal stenosis, foreign bodies, sinusitis. etc., the disease should be more common if it arose from these. Dr. Nichele, relying on his statistics, contends that, while scrofula is very common, this disease is rare; that if it depended on scrofula it should be sometimes bilateral, while it is practically always unilateral. The rapid cure also excludes scrofula; there is, moreover, only one case of recurrence reported in the literature, that of Massei.

As regards the bacteriology, the author points out the results obtained, and gives a list of the organisms found in the cases reported as true rhinitis caseosa. The constant and specific form of microbe, which could be regarded as the essential cause of the malady, is wanting. He sums up the etiology of the disease, a purulent secretion and an obstacle to its elimination. Having referred to the more recent cases of Cozzolino, Wagnier, Cimmino and others, he draws the following conclusions :

1. The small number of cases of rhinitis cascosa iera, with some exceptions, present the clinical features necessary for the disease according to the common theory. The rare exceptions leave room to doubt that the observations have been exact.

2. The rarity of the affection, the rapidity of cure and absence of recurrence exclude scrofula as a cause.

3. The disease cannot be regarded as microbic, because no specific microbe has been found.

He especially sets aside the Streptothrix alba of Guarnaccia, because it was found in a case of the so-called false disease, and even if it had been in one of the truc it does not matter, as it so closely resembles the filamentous forms seen in pseudo-rhinitis. Moreover, the Streptothrix alba inoculated has never produced the disease in man or animal.

James Donelan.

0stmann (Marbury).-Obliteration of the Tessels in the Nose by the Galicano-Cautery, as a Preliminary to Intranasal Operations.

"Deutsche Medicinische Wochenschrift," 1901, No. 14.

Ostmann recommends that the arterial supply of the nose should be stopped by the electro-cautery before operating on the nose. The loss of blood is reduced to a minimum, and the operation field remains clear.

Guild.

\section{LARYNX.}

Monselles, Salvadore.-Papilloma of the Larynx in Children and their Treatment. "Archiv. für Kinderheilkunde."

This is a lengthy paper of thirty pages, which gives a very full account of laryngeal papilloma. The pathology is fully discussed, with the help of quotations from various authors. The clinical history of some cases falling under his own observations is given. The various methods

1 Annal. di Laring. ed Otolog., 1900. 